\title{
Magnetic resonance imaging perfusion is associated with disease severity and activity in multiple sclerosis
}

Piotr Sowa (1, 2), Gro Owren Nygaard (3), Atle Bjørnerud (4, 5), Elisabeth Gulowsen Celius (3, 6), Hanne Finstad Harbo (2, 3), Mona Kristiansen Beyer (1, 7)

(1) Department of Radiology and Nuclear Medicine, Oslo University Hospital, Oslo, Norway

(2) Institute of Clinical Medicine, Faculty of Medicine, University of Oslo, Oslo, Norway

(3) Department of Neurology, Oslo University Hospital, Oslo, Norway

(4) Intervention Center, Oslo University Hospital, Oslo, Norway

(5) Department of Physics, University of Oslo, Oslo, Norway

(6) Institute of Health and Society, Faculty of Medicine, University of Oslo, Oslo, Norway

(7) Department of Life Sciences and Health, Oslo and Akershus University College of Applied Sciences, Oslo, Norway

\section{ABSTRACT}

\section{Purpose}

The utility of perfusion weighted imaging in multiple sclerosis (MS) is not well investigated. The purpose of this study was to compare baseline normalized perfusion measures in subgroups of newly diagnosed MS patients. We wanted to test the hypothesis that this method can differentiate between groups defined according to disease severity and disease activity at one year follow-up.

\section{Methods}

Baseline magnetic resonance imaging (MRI) including a dynamic susceptibility contrast perfusion sequence was performed on a 1.5 Tesla scanner in 66 patients newly diagnosed with relapsing-remitting MS. From the baseline MRI, cerebral blood flow (CBF), cerebral blood volume (CBV) and mean transit time (MTT) maps were generated. Normalized (n) perfusion values were calculated by dividing each perfusion parameter obtained in white matter lesions by the same parameter obtained in normal appearing white matter. Neurological examination was performed at baseline and at follow-up approximately one year later to establish the multiple sclerosis severity score (MSSS) and evidence of disease activity (EDA). 


\section{Results}

Baseline nMTT was lower in patients with MSSS>3.79 ( $\mathrm{p}=0.016)$, in patients with EDA $(\mathrm{p}=0.041)$ and in patients with both MSSS $>3.79$ and EDA $(\mathrm{p}=0.032)$ at one-year follow-up. Baseline nCBF and nCBV did not differ between these groups.

\section{Conclusions}

Lower baseline nMTT was associated with higher disease severity and with presence of disease activity one year later in newly diagnosed MS patients. Further longitudinal studies are needed to confirm whether baseline normalized perfusion measures can differentiate between disease severity and disease activity subgroups over time.

\section{Keywords}

Disease activity, Disease severity, Magnetic resonance imaging, Mean transit time, Multiple sclerosis, Perfusion weighted imaging

\section{Abbreviations}

$\mathrm{CBF}=$ cerebral blood flow; $\mathrm{CBV}=$ cerebral blood volume; $\mathrm{DMT}=$ disease modifying treatment; EDA = evidence of disease activity; EDSS = expanded disability status scale; MRI = magnetic resonance imaging; $\mathrm{MS}=$ multiple sclerosis; $\mathrm{MSSS}=$ multiple sclerosis severity score; MTT = mean transit time; $\mathrm{n}=$ normalized; NAWM = normal appearing white matter; NEDA = no evidence of disease activity; PVE = partial volume effect; $\mathrm{PWI}=$ perfusion weighted imaging; $\mathrm{WML}=$ white matter lesions

\section{INTRODUCTION}

Multiple sclerosis (MS) is an important cause of neurological disability in young adults [1]. The disease varies in terms of severity, and both disease progression and disease activity are difficult to predict at onset [2-5]. Imaging biomarkers that could help in early identification of patients at risk of developing a severe disease course would be a useful tool in clinical management. There have been several attempts to grade disease severity in MS and to identify prognostic parameters for benign and severe disease course, including volumetric measures 
[6-10]. To date, no such parameters have been validated. To assess disease severity in MS at a single time point the MS severity score (MSSS) can be used. MSSS describes disease severity as neurological disability in relation to disease duration, and has been proven useful for comparing groups of patients [11]. Disease activity can be assessed using the concept of evidence of disease activity (EDA), based on a composite of clinical and radiological measures. The concept was recently introduced in clinical trials where the absence of EDA no evidence of disease activity (NEDA), was used as an outcome measure in evaluation of disease modifying treatments (DMT) [12-14].

Magnetic resonance imaging (MRI) is an important tool in diagnosis and follow-up of MS patients [15-17]. Furthermore, advanced MRI has shown promising results in establishing prognosis and in disease monitoring in MS, but it is still not well explored and a need for new MRI markers has been highlighted [10]. One advanced MRI technique is dynamic susceptibility contrast perfusion weighted imaging (PWI) that enables measurement of cerebral blood flow (CBF), cerebral blood volume (CBV) and mean transit time (MTT) in brain tissue [18]. Due to known large technical and biological variations in these perfusion measures only normalized perfusion values can reliably be compared across individuals [19], calculated as the ratio of a perfusion measure from two different brain regions in the same subject. Such normalization has been extensively used in neurooncology [20, 21] and cerebral ischemic disease [22], while in MS it has been used e.g. for assessment of perfusion in lesions normalized to contralateral NAWM [23] or for analyzing the association between clinical data and perfusion in different brain regions normalized to hippocampi [24]. There is some literature describing PWI findings in relation to clinical data in patients with MS [24-27] but, to our knowledge, there are no previous reports exploring normalized perfusion measures in relation to disease severity and activity in MS.

This study is based on perfusion data from our previous baseline study [28] and on clinical data from approximately one-year follow-up [29]. We aimed to investigate whether there are differences in normalized perfusion measures between clinical subgroups of newly diagnosed MS patients, defined according to disease severity and disease activity. We hypothesized that patients with higher severity (higher MSSS scores), with active disease (presence of EDA) and with both higher severity and active disease one year later differ in their baseline normalized perfusion measures from patients with a more benign disease course. 


\section{MATERIALS AND METHODS}

\subsection{Subjects}

Sixty-six patients newly diagnosed with relapsing-remitting (RR) MS according to the revised 2010 McDonald criteria [17] were prospectively enrolled in this study. The patients were diagnosed with MS between January 2009 and October 2012, and recruited from an on-going longitudinal study at our institution [29]. All patients underwent a detailed neurological examination at baseline within $14.4 \pm 9.6$ months (range 1-34) from diagnosis. The baseline MRI scan was acquired within a week from the baseline neurological examination. The follow-up neurological examination and the follow-up MRI scan were repeated approximately one year later (on average $14 \pm 1.7$ months). The inclusion criteria at baseline were: age 1850 years, no more than three years since MS diagnosis, at least six weeks since the last relapse or steroid treatment, no prior neurological, neurovascular or psychiatric disease, no substantial head injury or drug abuse. The exclusion criteria were: pregnancy or breastfeeding, previous adverse reaction to gadolinium injection and inadequate image quality on MRI scans. The following demographical and clinical measures were obtained in all patients at baseline: age, sex, age at disease onset, time since diagnosis, disease duration, neurological disability as measured by expanded disability status scale (EDSS) [30], number of relapses and use of DMT. MSSS was calculated based on EDSS and disease duration. At baseline, 68\% of the patients received first-line DMT, $14 \%$ received second line DMT and 18\% no DMT. At follow-up the EDSS was reassessed, the MSSS was calculated, the number of new relapses was recorded and the EDA/NEDA status was determined based on the clinical and radiological progression as previously published [29]. The clinical data could not be obtained in one patient at follow-up. The characteristics of the patient cohort are presented in table 1A.

Table 1. Characteristics of the patient $\operatorname{cohort}^{\mathrm{a}}, \mathrm{n}=\mathbf{6 6}$

\section{A. Demographic and clinical characteristics}

Age at baseline, years

Female:male ratio

Age at disease onset, years

Time since diagnosis at baseline, months

Disease duration at baseline, months

Time between baseline and follow-up, months
$34.9 \pm 7.2$

2:1

$32.6 \pm 6.7$

$14.4 \pm 9.6$

$21(10-33)$

$14.0 \pm 1.7$ 
Neurological disability by EDSS at follow-up ( $\mathrm{n}=65$ )

$2.0(1.5-2.5)$

Disease severity by MSSS at follow-up ( $n=65$ )

$4.22 \pm 1.99$

Nr of patients with EDA at follow-up $(n=65)$

$30(46 \%)$

Patients by disease modifying treatment ${ }^{\mathrm{b}}$

no treatment

$12(18 \%)$

first line treatment

45 (68\%)

second line treatment

$9(14 \%)$

\section{B. Imaging characteristics}

Normalized perfusion measures

\begin{tabular}{ll} 
nCBF $^{\mathrm{c}}$ & $0.82 \pm 0.20$ \\
nCBV $^{\mathrm{c}}$ & $1.02 \pm 0.30$ \\
nMTT $^{\mathrm{c}}$ & $1.27 \pm 0.15$ \\
Intracranial volume, ml $^{\text {Brain volume }}{ }^{\mathrm{d}}$ & $1445 \pm 123$ \\
Grey matter volume $^{\mathrm{d}}$ & $0.81 \pm 0.02$ \\
White matter volume $^{\mathrm{d}}$ & $0.42 \pm 0.02$ \\
WML volume (lesion load), ml $^{\text {fraction (\%) of white matter volume }}$ & $0.38 \pm 0.01$ \\
fraction (\%) of intracranial volume $^{\text {Number of WML }}{ }^{\mathrm{e}}$ per patient & $5.3(2.9-7.0)$ \\
total number of WML in whole cohort $_{\text {Contrast enhancing WML }}$ & $0.99 \pm 0.53$ \\
number of patients with enhancing WML & $0.38 \pm 0.20$ \\
number of enhancing WML per patient & $20.4 \pm 13.7$ \\
total number of enhancing WML in cohort & 1347 \\
\hline
\end{tabular}

EDA: Evidence of disease activity; EDSS: expanded disability status scale; MRI: magnetic resonance imaging; MS: multiple sclerosis; MSSS: multiple sclerosis severity scale; nCBF: normalized cerebral blood flow; nCBV: normalized cerebral blood volume; nMTT: normalized mean transit time; WML: white matter lesions.

${ }^{a}$ Data are $n(\%)$, mean \pm standard deviation, or median (interquartile range).

${ }^{\mathrm{b}}$ Disease modifying treatment: first line: interferon, glatiramer acetate, teriflunomide, dimethylfumarate; second line: natalizumab, fingolimod, alemtuzumab.

${ }^{\mathrm{c}}$ Normalized perfusion measures calculated as perfusion parameters obtained in WML divided by the same parameters obtained in normal appearing white matter.

${ }^{\mathrm{d}}$ Normalized to intracranial volume.

e Based on manually corrected WML mask co-registered to perfusion maps. 


\subsection{Image acquisition}

MRI scanning was performed as baseline on the same 1.5 Tesla scanner (Avanto, Siemens Medical, Erlangen, Germany) equipped with a 12-channel head coil. The following sequences were acquired:

(a) Sagittal 3D T1 MPRAGE (FOV: 240 x 240 mm; slice thickness: $1.2 \mathrm{~mm}$; voxel size: $1.3 \mathrm{x}$ 1.3 x 1.2 mm; TR: 2400 ms; TE: 3.61 ms; TI: 1000 ms; flip angle: 8;

(b) Pre-contrast sagittal 3D FLAIR (FOV: 260 x 260 mm; slice thickness: $1 \mathrm{~mm}$; voxel size: 1 x 1 x 1 mm; TR: 6000 ms; TE: 333 ms; TI: 2200 ms;

(c) Dynamic susceptibility contrast perfusion sequence (19 axial slices; FOV: 230 x 230 mm; slice thickness: 5 mm; voxel size: 1.8 x 1.8 x 5 mm; TR: 1400 ms; TE: 30 ms; flip angle: $90^{\circ}$ ). I.v. contrast agent (Dotarem, Laboratoire Guerbet, Paris, France) was administered at a dose $0.2 \mathrm{ml} / \mathrm{kg}$ and injection rate $5 \mathrm{ml} / \mathrm{sec}$.;

(d) Post-contrast T1 MPRAGE, with parameters identical to those of pre-contrast 3D T1, acquired approximately 7 minutes after contrast agent injection following the PWI acquisition.

\subsection{Image processing}

Details concerning image processing are given in a previous perfusion study at our institution that used the same patient material [28]. The perfusion data were processed with the nordicICE software package (www.nordicneurolab.com), resulting in CBF, CBV and MTT perfusion parametric maps (figure 1). A population based average arterial input function was used in the perfusion analysis. Correction for partial volume effects (PVE) was not specifically performed due to lack of established methods for this purpose. Proper PVE correction would require knowledge of the point spread function of the perfusion sequence [31], which is not easily obtainable for echo planar imaging sequences. We attempted to reduce PVE by down-sampling the high-resolution lesion mask to perfusion space, thereby avoiding any detrimental re-slicing of the low-resolution perfusion images. In the perfusion analysis, the parameters were adjusted to minimize the influence of the residual leakage. Leakage correction was performed using an established algorithm [32]. The algorithm uses linear fitting to estimate the T1 contamination caused by contrast agent extravasation, and by removing the leakage term it allows generation of corrected perfusion maps. 
Figure 1. Typical perfusion maps in a sample patient.

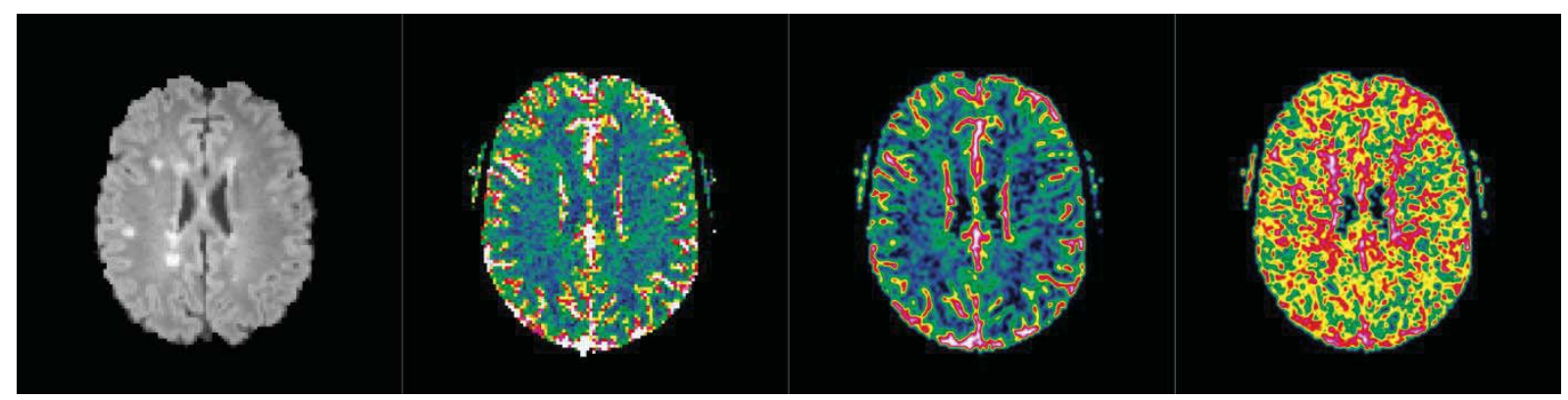

From the left: co-registered FLAIR series and CBF, CBV and MTT maps.

FLAIR: fluid-attenuated inversion recovery; CBF: cerebral blood flow, CBV: cerebral blood volume; MTT: mean transit time.

Binary masks of WM and GM were created from the volumetric T1 series using the Matlabbased Statistical Parametric Mapping toolbox (SPM8; http://www.fil.ion.ucl.ac.uk/spm). Volumetric measures and white matter lesions (WML) masks were generated automatically from the 3D T1 and FLAIR series using the CASCADE software (ki.se/en/nvs/cascade, Karolinska Institute, Stockholm, Sweden) [33]. The volumetric measures were normalized to intracranial volume. The structural series and the binary masks were co-registered to the perfusion maps using SPM8. The co-registered WML masks were visually inspected and edited in nordicICE software by a neuroradiologist to control for errors in the automatic lesion detection. The editing was performed by adding the automatically generated lesion segments as overlays on anatomical FLAIR series, which allowed adding and removing pixels from the WML mask. This correction provided quality control for the final WML masks. Dice similarity coefficient was calculated for the original and the edited WML masks. Region-ofinterest analysis was performed to extract perfusion parameters corresponding to the WML and NAWM masks. Normalized (n) perfusion measures were calculated by dividing each perfusion parameter obtained from the whole volume of WML by the same parameter obtained from the whole volume of NAWM. As a result, nCBF, nCBV and nMTT values were calculated for each patient. Normalization to the whole NAWM was chosen due to easy reproducibility and availability of whole WM segmentation methods today, and because previous reports showed that changes in NAWM in MS are general and found in both infraand supratentorial WM with diffusion tensor imaging [34]. Figure 2 shows WML and NAWM masks overlaid on a MTT perfusion map in a sample patient. In addition, the coregistered WML masks were post-processed using in-house software developed in Matlab R2012a (MathWorks, Natick, MA, USA) to obtain lesion count. 
Figure 2. FLAIR series, WM and WML masks and MTT perfusion map in a patient.

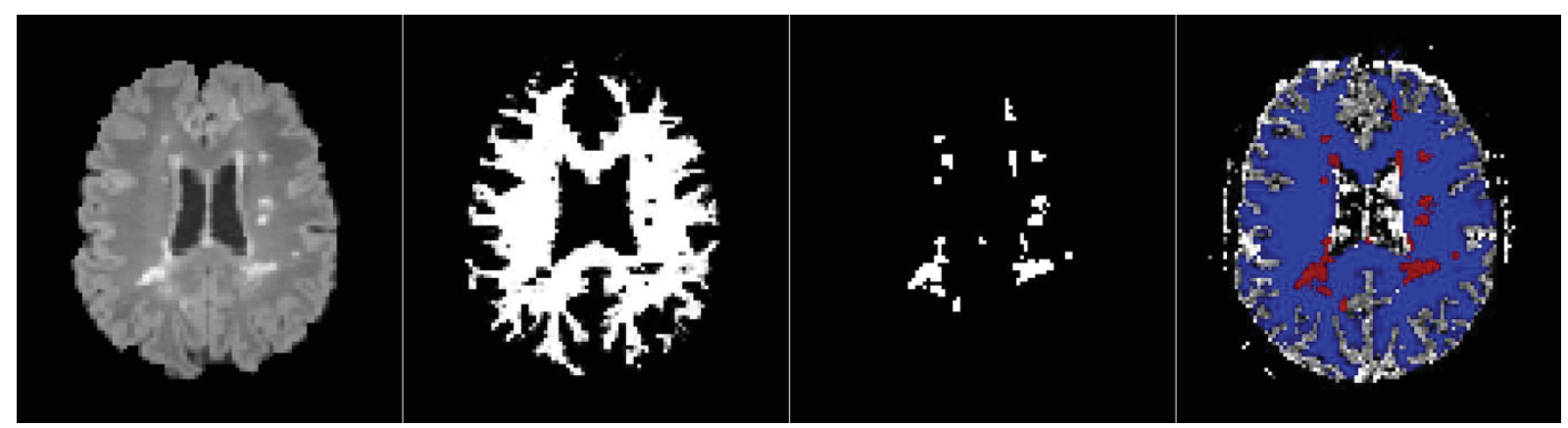

Normalized perfusion measures were calculated by dividing each perfusion parameter obtained in WML (red) by the same parameter obtained in NAWM (blue), using WM and WML masks. Here we see from the left: FLAIR series, WM mask, WML mask, and MTT perfusion map with overlaid WM and WML masks. Different patient than in figure 1.

FLAIR: fluid-attenuated inversion recovery; MTT: mean transit time; NAWM: normal appearing white matter; WM: white matter; WML: white matter lesions

There were only seven contrast-enhancing lesions in our material (one lesion each in seven patients), probably due to the fact that the patients were clinically stable when they came for scanning. In our study we wanted to focus on perfusion properties of the whole volume of lesions, but a separate analysis of the contrast enhancing lesions was performed by us previously [28]. The analysis showed no difference in perfusion parameters between the enhancing and the non-enhancing lesions $(\mathrm{p}=0.414, \mathrm{p}=0.904$ and $\mathrm{p}=0.332$ for $\mathrm{CBV}, \mathrm{CBF}$ and MTT, respectively). For this reason all lesions were included in the final analysis.

\subsection{Statistical analysis}

The Statistical Package for the Social Sciences software (SPSS v22, IBM, Chicago IL, USA) was used for all statistical analyses. Since the tested normalized perfusion parameters and volumetric measures were normally distributed in Kolmogorov-Smirnov normality test, the parametric one-way between-group analysis of covariance (ANCOVA) test was used for group analysis. Since we previously have shown that the EDA patients included in this study cohort used more first line treatment and less second line treatment than NEDA patients [29], all the group comparisons were performed with disease modifying treatment (DMT) as covariate, to control for possible influence of DMT on the results. Analyses of volumetric parameters were controlled for DMT and for age. All reported $p$-values are two-sided and the significance level was set to 0.05 . 


\section{RESULTS}

\subsection{Clinical characteristics}

Mean age of the patients at baseline was $34.9 \pm 7.2$ years, female: male ratio was 2:1, mean age at disease onset was $32.6 \pm 6.7$ years, median disease duration to baseline MRI was 21 months (range 3 -128) and mean time from diagnosis to baseline MRI was $14.4 \pm 9.6$ months (Table 1A). MSSS was $4.22 \pm 1.99(\mathrm{n}=65)$ at follow-up. The patients were divided in clinical groups according to disease severity measured by MSSS and according to EDA/NEDA status at follow-up. EDA status was found in 30 patients (46\%) patients.

\subsection{Imaging findings}

The imaging characteristics and volumetric measures are shown in details in table 1B. Mean nMTT was $1.27 \pm 0.15$. Mean brain volume normalized to intracranial volume was $0.81 \pm$ 0.20. Mean lesion count was $20 \pm 14$ per patient. Contrast enhancing lesions were observed in 7 patients only (one lesion in each patient), representing $0.5 \%$ of all 1347 lesions detected. Median total pixel number of the automatically generated WML mask (registered to perfusion maps) was 323 (range 128 - 1178), median total pixel number of the edited WML mask was 186 (range 24 - 1319) and mean Dice similarity coefficient between the masks was $0.51 \pm$ 0.24. Median absolute MTT values are given in Supplementary table 1.

\subsection{Group comparisons}

\subsubsection{Demographical and clinical characteristics of the groups}

The definitions of the groups are described in details below in each comparison. Proportion of females was different in NEDA group compared to EDA group and different in group with MSSS $\leq 3.79$ and NEDA compared to group with MSSS $>3.79$ and EDA. Mean age was different in group with MSSS $\leq 3.79$ compared to group with MSSS>3.79 (table 2). 
Table 2. Baseline characteristics of the groups, $\mathbf{n}=65$.

\begin{tabular}{|c|c|c|c|c|c|c|}
\hline & \multicolumn{6}{|c|}{ Groups defined according to } \\
\hline & \multicolumn{2}{|c|}{ Disease severity } & \multicolumn{2}{|c|}{ Disease activity } & \multicolumn{2}{|c|}{ Disease severity \& activity } \\
\hline & $\begin{array}{c}\text { MSSS } \leq 3.79 \\
n=29\end{array}$ & $\begin{array}{c}\text { MSSS }>3.79 \\
n=36\end{array}$ & $\begin{array}{l}\text { NEDA } \\
n=35\end{array}$ & $\begin{array}{l}\text { EDA } \\
\mathrm{n}=30\end{array}$ & $\begin{array}{c}\text { MSSS } \leq 3.79 \\
\& \text { NEDA } \\
n=44\end{array}$ & $\begin{array}{c}\text { MSSS }>3.79 \\
\text { \& EDA } \\
n=21\end{array}$ \\
\hline Female & $23(79 \%)$ & $22(61 \%)$ & $31(89 \%)^{a}$ & $14(47 \%)^{\mathrm{a}}$ & $36(81 \%)^{b}$ & $9(43 \%)^{b}$ \\
\hline Age, years & $32.6 \pm 6.5^{c}$ & $36.6 \pm 6.9^{c}$ & $35.3 \pm 7.5$ & $34 \pm 6.4$ & $34.3 \pm 7.5$ & $35.6 \pm 5.7$ \\
\hline $\begin{array}{l}\text { Disease } \\
\text { duration, years }\end{array}$ & $1.9(1.2-4)$ & $1.4(0.8-2.6)$ & $1.9(1.3-2.8)$ & $1.2(0.7-2.5)$ & $1.9(1.1-3)$ & $1.1(0.8-2.2)$ \\
\hline
\end{tabular}

EDA: evidence of disease activity; MSSS: multiple sclerosis severity score; NEDA: no evidence of disease activity.

Data are $n(\%)$, mean \pm standard deviation, or median (interquartile range).

$p$-values $<0.05$ from $\chi 2$-tests, independent samples t-tests or Mann-Whitney U tests (as appropriate) are indicated as follows:

${ }^{a}$ Proportion of females was different in NEDA group compared to EDA group;

${ }^{\mathrm{b}}$ Proportion of females was different in MSSS $\leq 3.79$ and NEDA group compared to MSSS>3.79 and EDA group;

${ }^{\mathrm{c}}$ Mean age was different in MSSS $\leq 3.79$ group compared to MSSS>3.79 group.

\subsubsection{Comparison of groups defined according to disease severity by MSSS at follow-up}

Since benign MS is usually defined as EDSS of three or less at least 10 years from disease onset [6-8], we defined the group with higher disease severity as patients with MSSS>3.79 (equivalent to EDSS>3.0 at 10 years of disease duration) and lower severity group as patients with MSSS $\leq 3.79$ (equivalent to EDSS $\leq 3.0$ at 10 years of disease duration). The nMTT was lower (ANCOVA: $\left.p=0.016, \mathrm{~F}(1,62)=6.12, \eta^{2}=0.09\right)$ in patients with higher severity $(\mathrm{n}=36)$ compared to patients with lower severity $(n=29)$ while nCBF and nCBV did not differ between the groups (table 3A and figure 3A). The baseline whole brain volume was lower (ANCOVA: $p=0.043, \mathrm{~F}(1,62)=4.28, \eta^{2}=0.07$ ) in patients with higher severity while $\mathrm{GM}$ and WM volumes showed no difference between the MSSS groups (table 4A). 
Table 3. Baseline normalized perfusion measures in patient groups defined according to disease severity by MSSS (A), disease activity by EDA/NEDA status (B) and both disease severity and activity (C) at one-year follow-up, $n=65$.

A. Groups defined according to disease severity by MSSS

\begin{tabular}{lcccc}
\hline & MSSS $\leq 3.79$ & MSSS $>3.79$ & F, partial $\eta^{2}$ & $p$-value \\
& $\mathrm{n}=29$ & $\mathrm{n}=36$ & & 0.896 \\
$\mathrm{nCBF}$ & $0.82 \pm 0.18$ & $0.81 \pm 0.22$ & $0.02,<0.01$ & 0.697 \\
nCBV & $1.05 \pm 0.26$ & $1.00 \pm 0.33$ & $0.15,<0.01$ & $\mathbf{0 . 0 1 6}$ \\
nMTT & $1.32 \pm 0.16$ & $1.23 \pm 0.12$ & $6.12,0.09$ & \\
\hline
\end{tabular}

B. Groups defined according to disease activity by EDA/NEDA status

\begin{tabular}{lcccc}
\hline & NEDA & EDA & F, partial $\eta^{2}$ & $p$-value ${ }^{\mathrm{a}}$ \\
& $\mathrm{n}=35$ & $\mathrm{n}=30$ & & 0.965 \\
nCBF & $0.81 \pm 0.16$ & $0.82 \pm 0.24$ & $<0.01,<0.01$ & 0.575 \\
nCBV & $1.03 \pm 0.23$ & $1.01 \pm 0.36$ & $0.32,<0.01$ & $\mathbf{0 . 0 4 1}$ \\
\hline
\end{tabular}

C. Groups defined according to both disease severity by MSSS and disease activity by EDA/NEDA status

\begin{tabular}{lcccc}
\hline & MSSS $\leq 3.79$ & MSSS $>3.79$ & & \\
& and NEDA & and EDA & F, partial $\eta^{2}$ & $p$-value ${ }^{\text {a }}$ \\
nCBF & $\mathrm{n}=44$ & $\mathrm{n}=21$ & & 0.953 \\
nCBV & $0.81 \pm 0.17$ & $0.82 \pm 0.25$ & $<0.01,<0.01$ & 0.511 \\
nMTT & $1.03 \pm 0.26$ & $1.00 \pm 0.37$ & $0.44,<0.01$ & $\mathbf{0 . 0 3 2}$ \\
\hline
\end{tabular}

EDA: evidence of disease activity; MSSS: multiple sclerosis severity score; nCBF: normalized cerebral blood flow; nCBV: normalized cerebral blood volume; NEDA: no evidence of disease activity; nMTT: normalized mean transit time.

${ }^{a}$ One-way analysis of covariance (ANCOVA), controlled for disease modifying treatment. 
Figure 3. Baseline nMTT shown in groups defined according to disease severity by MSSS (A), disease activity by EDA/NEDA status (B) and both disease severity and activity (C), n=65.

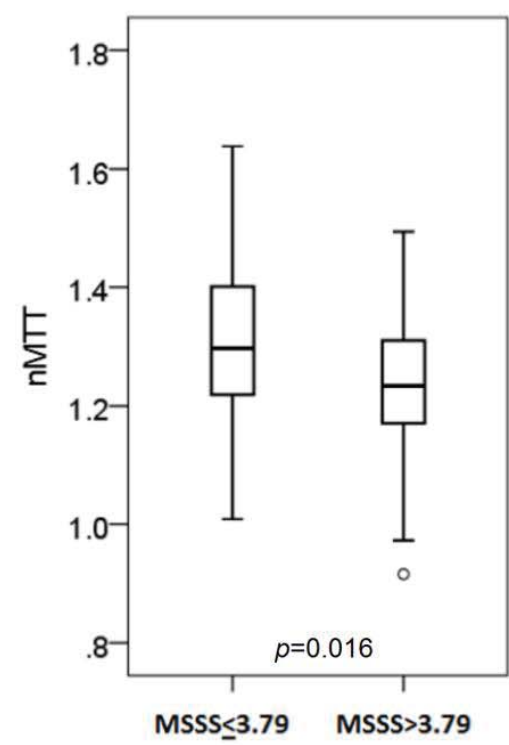

A

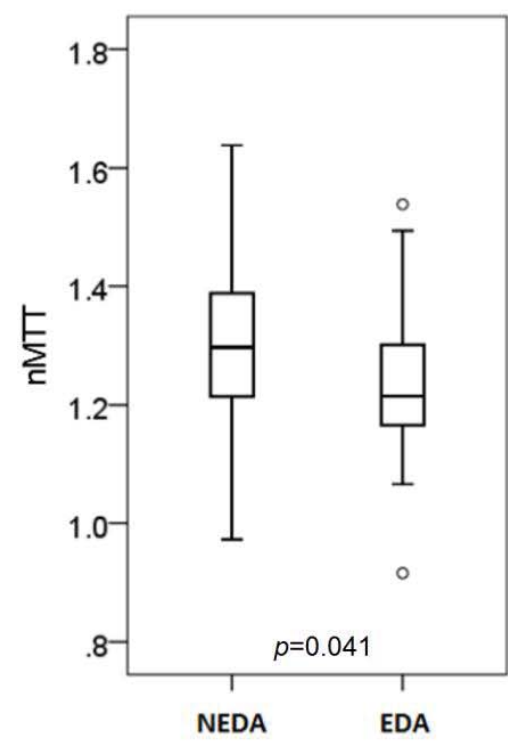

B

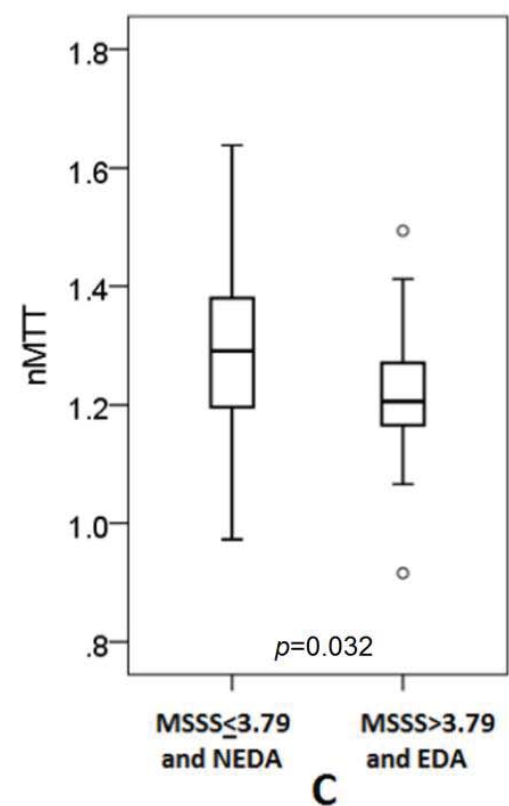

C

Baseline nMTT was significantly lower $(p=0.016)$ in patients with higher severity of MSSS>3.79 (n=36) compared to patients with lower severity of MSSS $\leq 3.79(n=29)(A)$, lower $(p=0.041)$ in patients with EDA $(n=30)$ compared to patients with NEDA $(n=35)(B)$, and lower $(p=0.032)$ in patients with MSSS $>3.79$ and EDA ( $n=21)$ compared to patients with MSSS $\leq 3.79$ and NEDA $(n=44)(C)$ at one-year follow-up, controlled for disease modifying treatment. Rings are outliers.

EDA: evidence of disease activity; MSSS: multiple sclerosis severity score; nCBF: normalized cerebral blood flow; nCBV: normalized cerebral blood volume; NEDA: no evidence of disease activity; nMTT: normalized mean transit time. 
Table 4. Baseline volumetric data in patient groups defined according to disease severity by MSSS (A), disease activity by EDA/NEDA status (B) and both disease severity and activity (C) at one-year follow-up, $n=65$.

A. Groups defined according to disease severity by MSSS

\begin{tabular}{lcccc}
\hline & MSSS $\leq 3.79$ & MSSS $>3.79$ & F, partial $\eta^{2}$ & $p$-value \\
& $\mathrm{n}=29$ & $\mathrm{n}=36$ & $4.28,0.07$ & $\mathbf{0 . 0 4 3}$ \\
Brain volume $^{\mathrm{b}}$ & $0.82 \pm 0.01$ & $0.80 \pm 0.02$ & $1.11,0.02$ & 0.297 \\
GM volume $^{\mathrm{b}}$ & $0.44 \pm 0.01$ & $0.43 \pm 0.02$ & $2.83,0.04$ & 0.097 \\
WM volume $^{\mathrm{b}}$ & $0.38 \pm 0.01$ & $0.38 \pm 0.01$ &
\end{tabular}

B. Groups defined according to disease activity by EDA/NEDA status

\begin{tabular}{lcccc}
\hline & NEDA & EDA & F, partial $\eta^{2}$ & $p$-value ${ }^{\mathrm{a}}$ \\
& $\mathrm{n}=35$ & $\mathrm{n}=30$ & $1.08,0.02$ & 0.304 \\
Brain volume $^{\mathrm{b}}$ & $0.81 \pm 0.02$ & $0.81 \pm 0.02$ & $1.49,0.02$ & 0.226 \\
GM volume $^{\mathrm{b}}$ & $0.43 \pm 0.02$ & $0.43 \pm 0.01$ & $<0.01,<0.01$ & 0.950 \\
WM volume $^{\mathrm{b}}$ & $0.38 \pm 0.01$ & $0.38 \pm 0.01$ & \\
\hline
\end{tabular}

C. Groups defined according to both disease severity by MSSS and disease activity by EDA/NEDA status

\begin{tabular}{lcccc}
\hline & MSSS $\leq 3.79$ & MSSS $>3.79$ & & \\
& and NEDA & and EDA & F, partial $\eta^{2}$ & $p$-value \\
Brain volume $^{\mathrm{b}}$ & $0.81 \pm 0.02$ & $0.80 \pm 0.02$ & $2.61,0.04$ & 0.111 \\
GM volume $^{\mathrm{b}}$ & $0.43 \pm 0.02$ & $0.43 \pm 0.01$ & $1.56,0.03$ & 0.216 \\
WM volume $^{\mathrm{b}}$ & $0.38 \pm 0.01$ & $0.38 \pm 0.01$ & $0.57,0.01$ & 0.455 \\
\hline
\end{tabular}

EDA: evidence of disease activity; GM: grey matter; MSSS: multiple sclerosis severity score; NEDA: no evidence of disease activity; WM: white matter.

${ }^{a}$ One-way analysis of covariance (ANCOVA), controlled for age and disease modifying treatment.

${ }^{\mathrm{b}}$ Volumes normalized to intracranial volume.

3.3.3 Comparison of groups defined according to disease activity by EDA/NEDA status.

The participants were grouped according to disease activity as defined by their EDA/NEDA status established at one-year follow-up in a previous clinical study in the same cohort [29] (EDA was defined as a presence of at least one of the following: disability progression, new relapse(s) or radiological progression). Only nMTT differed depending on the EDA/NEDA status: it was lower (ANCOVA: $\left.p=0.041, \mathrm{~F}(1,62)=4.35, \eta^{2}=0.07\right)$ in the EDA group $(\mathrm{n}=30)$ compared to the NEDA group $(n=35)$, while $n C B F$ and $n C B V$ were similar in both groups 
(table 3B and figure 3B). The volumetric measurements showed no difference in this comparison (table 4B).

3.3.4 Comparison of groups defined according to disease severity by both MSSS and EDA/NEDA status at follow-up.

In this analysis the patients were divided in two groups according both to their disease severity as measured by MSSS and disease activity defined by EDA/NEDA status. One group was defined as patients with more severe disease course (follow-up MSSS>3.79 and EDA) and the other group as patients with more benign disease course (follow-up MSSS $\leq 3.79$ and NEDA). The nMTT was significantly lower in the more severe group $(n=21)$ compared to the more benign group $(n=44)$ after controlling for age and DMT (ANCOVA: $p=0.032$, $\mathrm{F}(1,62)=4.81, \eta^{2}=0.07$ ) while $\mathrm{nCBF}$ and $\mathrm{nCBV}$ were similar (table 3C and figure 3C). Hence, nMTT was lower in the less benign group regardless of whether patients were grouped according to MSSS and EDA/NEDA status alone or according to both. The volumetric measurements were similar in this comparison (table 4C).

\section{DISCUSSION}

In this prospective longitudinal study of newly diagnosed MS patients we found that baseline nMTT was significantly lower in patients with higher disease severity as assessed by MSSS at one-year follow-up. These results suggest that the baseline microvascular properties of MS lesions (related to NAWM) in newly diagnosed MS patients differ with future disease development. Previous studies have revealed heterogeneity of histopathological changes in MS [35-37]. There is ongoing debate about different mechanisms of demyelination [36, 38]. Perivascular T cell infiltration and microglia activation are widespread in many MS patients as well as scattered parenchymal $\mathrm{T}$ cell infiltration, also in the chronic disease phase [39]. These findings are also supported in a previous study by Adams et al. [40] who found lymphocytic perivascular infiltration, lesion hypercellularity and macrophage infiltration in MS lesions. In general little is reported about vascular changes associated with MS lesions. Lesions in MS tend to be in perivenular locations [41]. Lassmann et al. [39] reported that disturbances of the microcirculation due to focal edema within inflammatory lesions, inflammatory reaction of the vessel wall resulting in microvascular thrombosis, or endothelial damage by T-lymphocytes are possible mechanisms of demyelination in some patients. An 
increased density of microvessels has also been reported by the authors. All these factors may influence perfusion parameters in MS patients. It is therefore plausible that vascular properties of both the WML and NAWM in MS patients can vary across individuals depending on vascular involvement in the pathological changes. In our previous perfusion study we reported reduced perfusion in WML compared to NAWM in MS patients [28]. Thus, lower nMTT in patients with higher disease severity can be interpreted as less reduced (or relatively increased) WML perfusion, which might be caused by different patterns of vascular or perivascular affection in these patients, compared to the patient group with lower disability. Alternatively, this finding could also indicate predominating changes in NAWM (relatively reduced perfusion in NAWM compared to WML).

Furthermore, the nMTT differed significantly between groups defined according to disease activity: it was lower in the EDA group where disease activity was present at one-year followup, and in the group defined as patients with both higher disease severity and EDA at followup. The fact that higher severity (determined by MSSS) and presence of activity (determined by EDA/NEDA status) were both associated with lower nMTT suggests a similar biological explanation. Interestingly, observed variations in nMTT in our study were not accompanied by a corresponding variation in $\mathrm{nCBV}$ or $\mathrm{nCBF}$. This may seem surprising, given that MTT is defined as the ratio CBV/CBF [18]. However, nCBV and nCBF are estimated globally from the ratio of mean values in WML and NAWM whereas MTT is estimated pixel-wise prior to normalization. Hence, nMTT may reveal variations, not reflected in the global nCBV and nCBF measures.

Although only normalized values should be compared between patient groups, we performed additional analysis of absolute MTT values in NAWM and WML between the groups to see which region, WML or NAWM influences the normalized MTT values. This analysis suggested that the nMTT results between MSSS groups are driven mainly by changes in WML ( $p=0.048$ ), and between the EDA/NEDA groups are driven mainly by changes in NAWM $(p=0.025)$. The detailed absolute MTT values in the groups are shown in supplementary Table 1 . These results should be interpreted with caution as we cannot estimate the influence of physiologically and technically related variations.

The baseline total brain volume was significantly lower in patients with higher severity. This finding is partly in line with previous research reports where whole brain atrophy [42] and 
baseline GM volumes [43, 44] were associated with disease severity in MS. No volumetric measures showed significant difference between groups defined according to disease activity (determined by EDA/NEDA status) in our study. In our analyses the nMTT showed more significant results than atrophy measurements in all performed group comparisons.

Our finding of no difference in perfusion measures between enhancing and non-enhancing lesions may seem surprising but it is at least partly supported by a previous study from 2005 [23] where non-enhancing lesions referred to as class "two" were not distinguishable from enhancing lesions in their perfusion properties. In our material, because of a very low number of enhancing lesions (7 of all 1347 lesions detected) the contribution of these lesions to perfusion values would be negligible.

Dice similarity coefficient between the original and the edited WML masks was low (0.51 \pm 0.24) which confirms that modification of the original mask (i.e. visual inspection and editing) was necessary.

The strength of the study is that the patients underwent a detailed neurological examination as previously described [29, 45]. The patients were newly diagnosed and are followed longitudinally. The number of participants is relatively large compared to other published perfusion studies in MS which typically included not more than 45 subjects [25-27]. We used a semi-automated image processing method for creating binary masks; this is an advantage as it minimizes the user bias. The perfusion sequence is not time consuming and does not require more intravenous contrast than the standard dose routinely used in MS. Thus this sequence can easily be added to the scanning protocol without increasing scan length with more than two minutes. Our approach in the perfusion analysis (use of normalized perfusion measures, not absolute values) provides reliability for comparisons across subjects, which is also strength of our study.

The main limitation of the study is a short observation time which was 14 months on average. A longer follow-up time is interesting as it would increase the usefulness of the perfusion sequence if it proves to be associated with clinical findings even after 2-3 years or more. Another limitation is that the MRI acquisition was on a 1.5T scanner, this gives a lower signal to noise ratio compared to $3.0 \mathrm{~T}$ and may have influenced our results. Our method that required use of lesion segmentation and perfusion analysis may seem time-consuming, but 
these techniques are under constant development and even today they are not more complicated than atrophy measurements; moreover, some neuroradiology departments use perfusion techniques routinely, and are familiar with these analyses. Normalization to NAWM in a disease of the whole brain can be questioned, but since the cohort of this study consists of newly diagnosed patients who have not yet reached the progressive phase it can be assumed that pathological changes in NAWM would be subtle in these patients [37]. In addition, a newer perfusion study has suggested normal perfusion in NAWM in MS patients compared to healthy controls [46].

In conclusion, lower baseline nMTT was associated with higher disease severity and with presence of disease activity one year later in newly diagnosed MS patients. This study suggests that MRI perfusion parameters are promising as imaging biomarkers of disease severity and activity in MS, but there is a need for further longitudinal research.

\section{Funding}

This study was funded by South-Eastern Norway Regional Health Authority (grant nr 39569). MRI scans and clinical tests were performed within a previous project financed by the same institution (grant nr 2011059).

\section{Conflict of interest}

Piotr Sowa received speaker honoraria from Novartis, Genzyme and Biogen.

Gro O. Nygaard received unrestricted research grants from Novartis Norway and from the Odd Fellow's Foundation for Multiple Sclerosis Research.

Atle Bjørnerud is a consultant for NordicNeuroLab AS, Bergen, Norway.

Elisabeth G. Celius received support for travelling and speaker honoraria from Biogen, Genzyme, Merck, Novartis, Sanofi-Aventis and Teva, and received unrestricted research grants from Biogen, Genzyme and Novartis.

Hanne F. Harbo received an unrestricted research grant from Novartis, and support for travelling and speaker honoraria from Biogen, Novartis, Sanofi-Aventis and Teva.

Mona K. Beyer received speaker honoraria from Novartis and Biogen.

\section{Ethical approval}

All procedures involving human participants performed in this study were in accordance with the ethical standards of the institutional and national research committee (data inspectorate 
representative at the hospital and the Regional Committee for Medical and Health Research Ethics for South-Eastern Norway) and with the 1964 Helsinki declaration and its later amendments.

\section{Informed consent}

Written informed consent was obtained from all individual participants included in the study.

\section{Acknowledgments}

The authors would like to thank Paulina Due-Tønnessen, Soheil Damangir, Gabriela Spulber and Kyrre Emblem for assistance.

Supplementary Table 1. Baseline absolute MTT values in WML and NAWM in patient groups defined according to disease severity by MSSS (A), disease activity by EDA/NEDA status (B) and both disease severity and activity (C) at one-year follow-up, $\mathrm{n}=65$.

A. Groups defined according to disease severity by MSSS

\begin{tabular}{lcccc}
\hline & MSSS $\leq 3.79$ & MSSS $>3.79$ & Z, r & $p^{- \text {value }^{\mathrm{a}}}$ \\
& $\mathrm{n}=29$ & $\mathrm{n}=36$ & & $\mathbf{0 . 0 4 8}$ \\
MTT in WML $^{\mathrm{b}}$ & $4.60(2.77-5.87)$ & $4.32(2.64-8.12)$ & $-1.98,-0.25$ & 0.979 \\
\hline
\end{tabular}

B. Groups defined according to disease activity by EDA/NEDA status

\begin{tabular}{lcccc}
\hline & NEDA & EDA & Z, r & $p^{- \text {value }^{\mathrm{a}}}$ \\
& $\mathrm{n}=35$ & $\mathrm{n}=30$ & & 0.281 \\
MTT in WML $^{\mathrm{b}}$ & $4.28(2.64-5.87)$ & $4.47(2.77-8.12)$ & $-1.08,-0.13$ & $\mathbf{0 . 0 2 5}$ \\
\hline MTT in NAWM
\end{tabular}

C. Groups defined according to both disease severity by MSSS and disease activity by EDA/NEDA status

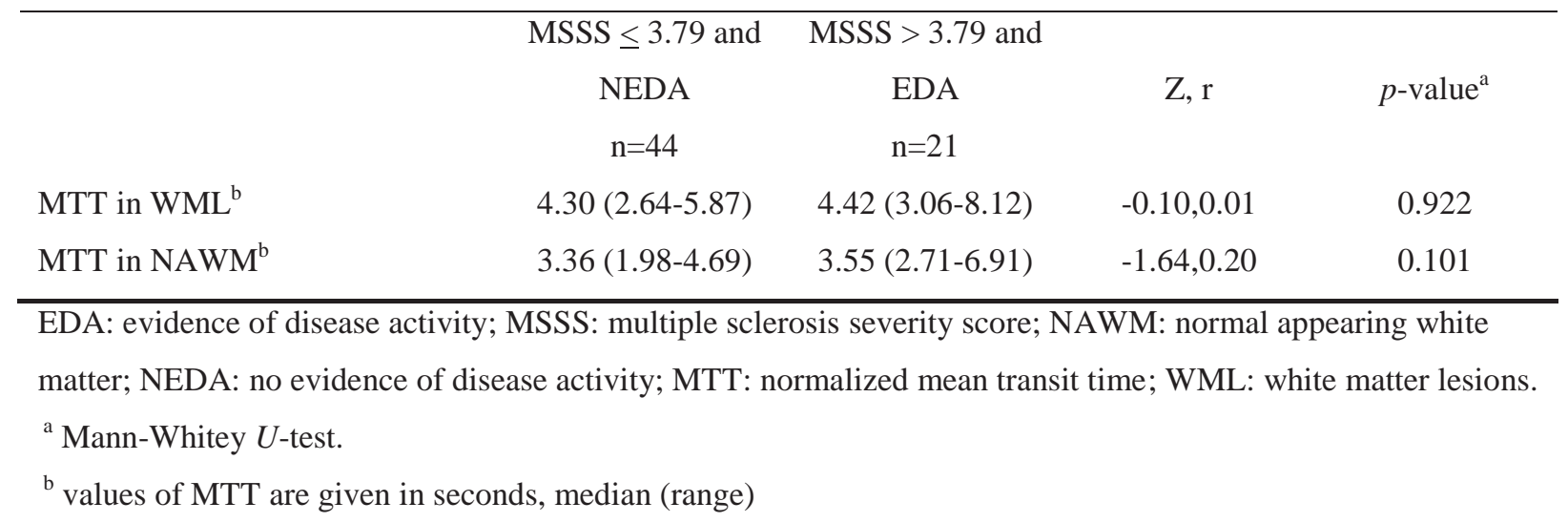




\section{References}

[1] Nicholas R, Rashid W. Multiple sclerosis. BMJ clinical evidence. 2012;2012.

[2] Rush CA, MacLean HJ, Freedman MS. Aggressive multiple sclerosis: proposed definition and treatment algorithm. Nat Rev Neurol. 2015;11:379-89.

[3] Trojano M, Liguori M, Bosco Zimatore G, Bugarini R, Avolio C, Paolicelli D, et al. Agerelated disability in multiple sclerosis. Ann Neurol. 2002;51:475-80.

[4] Confavreux C, Vukusic S. Natural history of multiple sclerosis: a unifying concept. Brain. 2006;129:606-16.

[5] Tremlett H, Zhao Y, Rieckmann P, Hutchinson M. New perspectives in the natural history of multiple sclerosis. Neurology. 2010;74:2004-15.

[6] Ramsaransing GS, De Keyser J. Predictive value of clinical characteristics for 'benign' multiple sclerosis. Eur J Neurol. 2007;14:885-9.

[7] Ramsaransing G, Maurits N, Zwanikken C, De Keyser J. Early prediction of a benign course of multiple sclerosis on clinical grounds: a systematic review. Mult Scler. 2001;7:3457.

[8] Hawkins SA, McDonnell GV. Benign multiple sclerosis? Clinical course, long term follow up, and assessment of prognostic factors. J Neurol Neurosurg Psychiatry. 1999;67:14852.

[9] De Stefano N, Airas L, Grigoriadis N, Mattle HP, O'Riordan J, Oreja-Guevara C, et al. Clinical relevance of brain volume measures in multiple sclerosis. CNS drugs. 2014;28:14756.

[10] Wattjes MP, Rovira A, Miller D, Yousry TA, Sormani MP, de Stefano MP, et al. Evidence-based guidelines: MAGNIMS consensus guidelines on the use of MRI in multiple sclerosis--establishing disease prognosis and monitoring patients. Nat Rev Neurol. 2015;11:597-606.

[11] Roxburgh RH, Seaman SR, Masterman T, Hensiek AE, Sawcer SJ, Vukusic S, et al. Multiple Sclerosis Severity Score: using disability and disease duration to rate disease severity. Neurology. 2005;64:1144-51.

[12] Rotstein DL, Healy BC, Malik MT, Chitnis T, Weiner HL. Evaluation of no evidence of disease activity in a 7-year longitudinal multiple sclerosis cohort. JAMA Neurol.

2015;72:152-8.

[13] Bevan CJ, Cree BA. Disease activity free status: a new end point for a new era in multiple sclerosis clinical research? JAMA Neurol. 2014;71:269-70.

[14] Giovannoni G, Cook S, Rammohan K, Rieckmann P, Sorensen PS, Vermersch P, et al. Sustained disease-activity-free status in patients with relapsing-remitting multiple sclerosis treated with cladribine tablets in the CLARITY study: a post-hoc and subgroup analysis. Lancet Neurol. 2011;10:329-37.

[15] Filippi M, Rocca MA, Ciccarelli O, De Stefano N, Evangelou N, Kappos L, et al. MRI criteria for the diagnosis of multiple sclerosis: MAGNIMS consensus guidelines. Lancet Neurol. 2016;15:292-303.

[16] Rovira A, Wattjes MP, Tintore M, Tur C, Yousry TA, Sormani MP, et al. Evidencebased guidelines: MAGNIMS consensus guidelines on the use of MRI in multiple sclerosisclinical implementation in the diagnostic process. Nat Rev Neurol. 2015;11:471-82.

[17] Polman CH, Reingold SC, Banwell B, Clanet M, Cohen JA, Filippi M, et al. Diagnostic criteria for multiple sclerosis: 2010 revisions to the McDonald criteria. Ann Neurol.

2011;69:292-302. 
[18] Ostergaard L. Principles of cerebral perfusion imaging by bolus tracking. J Magn Reson Imaging. 2005;22:710-7.

[19] Shin W, Horowitz S, Ragin A, Chen Y, Walker M, Carroll TJ. Quantitative cerebral perfusion using dynamic susceptibility contrast MRI: evaluation of reproducibility and ageand gender-dependence with fully automatic image postprocessing algorithm. Magn Reson Med. 2007;58:1232-41.

[20] Emblem KE, Bjornerud A. An automatic procedure for normalization of cerebral blood volume maps in dynamic susceptibility contrast-based glioma imaging. AJNR Am J

Neuroradiol. 2009;30:1929-32.

[21] Law M, Young RJ, Babb JS, Peccerelli N, Chheang S, Gruber ML, et al. Gliomas: predicting time to progression or survival with cerebral blood volume measurements at dynamic susceptibility-weighted contrast-enhanced perfusion MR imaging. Radiology. 2008;247:490-8.

[22] Copen WA, Schaefer PW, Wu O. MR perfusion imaging in acute ischemic stroke. Neuroimaging Clin N Am. 2011;21:259-83, x.

[23] Ge Y, Law M, Johnson G, Herbert J, Babb JS, Mannon LJ, et al. Dynamic susceptibility contrast perfusion MR imaging of multiple sclerosis lesions: characterizing hemodynamic impairment and inflammatory activity. AJNR Am J Neuroradiol. 2005;26:1539-47.

[24] Papadaki EZ, Simos PG, Panou T, Mastorodemos VC, Maris TG, Karantanas AH, et al. Hemodynamic evidence linking cognitive deficits in clinically isolated syndrome to regional brain inflammation. Eur J Neurol. 2014;21:499-505.

[25] Papadaki EZ, Simos PG, Mastorodemos VC, Panou T, Maris TG, Karantanas AH, et al. Regional MRI perfusion measures predict motor/executive function in patients with clinically isolated syndrome. Behav Neurol. 2014;2014:252419.

[26] Francis PL, Jakubovic R, O'Connor P, Zhang L, Eilaghi A, Lee L, et al. Robust perfusion deficits in cognitively impaired patients with secondary-progressive multiple sclerosis. AJNR Am J Neuroradiol. 2013;34:62-7.

[27] Inglese M, Adhya S, Johnson G, Babb JS, Miles L, Jaggi H, et al. Perfusion magnetic resonance imaging correlates of neuropsychological impairment in multiple sclerosis. J Cereb Blood Flow Metab. 2008;28:164-71.

[28] Sowa P, Bjornerud A, Nygaard GO, Damangir S, Spulber G, Celius EG, et al. Reduced perfusion in white matter lesions in multiple sclerosis. Eur J Radiol. 2015;84:2605-12.

[29] Nygaard GO, Celius EG, de Rodez Benavent SA, Sowa P, Gustavsen MW, Fjell AM, et al. A Longitudinal Study of Disability, Cognition and Gray Matter Atrophy in Early Multiple Sclerosis Patients According to Evidence of Disease Activity. PLoS One. 2015;10:e0135974. [30] Kurtzke JF. Rating neurologic impairment in multiple sclerosis: an expanded disability status scale (EDSS). Neurology. 1983;33:1444-52.

[31] Coello C, Willoch F, Selnes P, Gjerstad L, Fladby T, Skretting A. Correction of partial volume effect in (18)F-FDG PET brain studies using coregistered MR volumes: voxel based analysis of tracer uptake in the white matter. Neuroimage. 2013;72:183-92.

[32] Boxerman JL, Schmainda KM, Weisskoff RM. Relative cerebral blood volume maps corrected for contrast agent extravasation significantly correlate with glioma tumor grade, whereas uncorrected maps do not. AJNR Am J Neuroradiol. 2006;27:859-67.

[33] Damangir S, Westman E, Simmons A, Vrenken H, Wahlund L-O, Spulber G.

Reproducible segmentation of white matter hyperintensities using a new statistical definition. Magnetic Resonance Materials in Physics, Biology and Medicine. 2016:1-11.

[34] Ciccarelli O, Werring DJ, Wheeler-Kingshott CA, Barker GJ, Parker GJ, Thompson AJ, et al. Investigation of MS normal-appearing brain using diffusion tensor MRI with clinical correlations. Neurology. 2001;56:926-33. 
[35] Popescu BF, Pirko I, Lucchinetti CF. Pathology of multiple sclerosis: where do we stand? Continuum (Minneapolis, Minn). 2013;19:901-21.

[36] Stadelmann C, Wegner C, Bruck W. Inflammation, demyelination, and degeneration recent insights from MS pathology. Biochim Biophys Acta. 2011;1812:275-82.

[37] Kutzelnigg A, Lucchinetti CF, Stadelmann C, Bruck W, Rauschka H, Bergmann M, et al. Cortical demyelination and diffuse white matter injury in multiple sclerosis. Brain.

2005;128:2705-12.

[38] Lassmann H. Axonal injury in multiple sclerosis. J Neurol Neurosurg Psychiatry.

2003;74:695-7.

[39] Lassmann H. Hypoxia-like tissue injury as a component of multiple sclerosis lesions. J Neurol Sci. 2003;206:187-91.

[40] Adams CW, Poston RN, Buk SJ. Pathology, histochemistry and immunocytochemistry of lesions in acute multiple sclerosis. J Neurol Sci. 1989;92:291-306.

[41] Ge Y, Zohrabian VM, Grossman RI. 7T MRI: New Vision of Microvascular Abnormalities in Multiple Sclerosis. Arch Neurol. 2008;65:812-6.

[42] Popescu V, Agosta F, Hulst HE, Sluimer IC, Knol DL, Sormani MP, et al. Brain atrophy and lesion load predict long term disability in multiple sclerosis. J Neurol Neurosurg Psychiatry. 2013;84:1082-91.

[43] Lavorgna L, Bonavita S, Ippolito D, Lanzillo R, Salemi G, Patti F, et al. Clinical and magnetic resonance imaging predictors of disease progression in multiple sclerosis: a nineyear follow-up study. Mult Scler. 2014;20:220-6.

[44] Filippi M, Preziosa P, Copetti M, Riccitelli G, Horsfield MA, Martinelli V, et al. Gray matter damage predicts the accumulation of disability 13 years later in MS. Neurology.

2013;81:1759-67.

[45] Nygaard GO, Walhovd KB, Sowa P, Chepkoech JL, Bjornerud A, Due-Tonnessen P, et al. Cortical thickness and surface area relate to specific symptoms in early relapsing-remitting multiple sclerosis. Mult Scler. 2015;21:402-14.

[46] Ingrisch M, Sourbron S, Herberich S, Schneider MJ, Kumpfel T, Hohlfeld R, et al. Dynamic Contrast-Enhanced Magnetic Resonance Imaging Suggests Normal Perfusion in Normal-Appearing White Matter in Multiple Sclerosis. Invest Radiol. 2016. 